\title{
Boosting the uptake of the influenza vaccine in diabetics in general practice
}

\section{Vishaal Kumar Dovedi Anita Kanya lyer}

Barts and the London School of Medicine and Dentistry, Whitechapel, London, UK
Correspondence: Vishaal Kumar Dovedi Barts and the London School of Medicine and Dentistry, 4 Newark Street, Whitechapel, London, EI 2AT, UK Tel +44753858 4l 45

Email vishaaldovedi@aol.com
This article was published in the following Dove Press journal: Clinical Audit

Purpose: Diabetes has become the largest modern-day epidemic the developed world has ever seen. Approximately 3.5 million people in the UK live with the chronic condition. The primary aim of this audit was to assess the effect of increased patient notification on the uptake of the influenza vaccine in diabetic patients in an urban general practitioner practice. Our secondary aims involved identifying the intervention that increased the uptake by the greatest margin and addressing any barriers to uptake.

Patients and methods: The audit was carried out across a 7-week period. It involved carrying out various forms of patient notification including telephone calls and emails in a population of 393 diabetic patients who were yet to have the flu vaccine, after which the outcomes of their implementation were analyzed.

Results: Comparing pre-audit and post-audit figures, there was a $20 \%$ increase in the number of patients vaccinated against the flu across just a 7-week period. Calling patients with a prewritten script, proved to be the most effective form of patient notification.

Conclusion: The data demonstrate that notifying diabetic patients who require the flu vaccine is beneficial. This can have a wider purpose for other health care interventions in a variety of patient groups. Our findings have the potential to be relevant to all general practices looking to increase their percentage uptake of the flu vaccine in order to achieve or surpass Quality and Outcomes Framework targets. It is particularly pertinent for practices with diabetic patients or that face difficulties communicating with patients effectively.

Keywords: general practice, diabetes, influenza vaccine, public health

\section{Introduction}

Diabetes has become the largest modern-day epidemic the developed world has seen. Approximately 360 million people live with the serious life-long condition worldwide and numbers are expected to rise exponentially by 2030 to 552 million people. ${ }^{1}$ In the UK, the figures are just as astounding with 3.5 million diabetics of which $95 \%$ are as a result of type 2 diabetes. ${ }^{2}$ London has staggeringly high suspected rates of people with diabetes at over 350,000 people, and in the East London borough of Newham the rates are as high as 20,800 with 1,600 new diagnoses every year. It is estimated that currently $10 \%$ of the UK National Health Service (NHS) funds goes toward diabetes and all its associated complications. ${ }^{3}$

Diabetes is associated with various complications including more frequent and severe forms of infection. The high glucose levels in a diabetic encourage immune dysfunction such as poor neutrophil function, suppressed antioxidant system, and humoral 
immunity, ${ }^{4}$ thereby putting the patients in an immunocompromised state, increasing their susceptibility to infections.

The influenza virus is particularly common in the diabetic population. It has been estimated that people with diabetes are six times more likely to be hospitalized during influenza epidemics compared to their nondiabetic counterparts. ${ }^{5}$ With their vulnerability to such a widespread infection, it is no wonder that the WHO has recommended that all diabetics be vaccinated against the influenza virus.

The WHO identifies the strains of influenza virus that are most likely to be circulating around the winter months in the northern hemisphere. The three strain types found in the vaccine are A/H1N1, A/H3N2, and Influenza B. These viruses are cultured, killed, and purified to form the vaccine. When the deactivated version of these viruses enter the body, the immune system is triggered to form a response against the foreign antigens on the viruses. After fighting off these viruses, the immune system forms memory cells to help fight against the same viruses should they enter the body again. This protects the patient from the flu (caused by these particular strains) for the duration of the winter months of that year. Due to antigenic shift, new influenza virus strains form by the next winter season; therefore, WHO recommends annual flu vaccines for eligible patients. ${ }^{6}$

The flu vaccine is given intramuscularly and is a free service on the NHS for patients with chronic conditions such as diabetes. It can be administered at general practitioner surgeries, local pharmacies, and in hospitals. This makes it an easy and accessible disease prevention scheme that has improved public health since its inception in the $1960 \mathrm{~s}^{7}$

\section{Objectives}

The primary objective of this audit was to assess the effect of increased patient notification on the uptake of the flu vaccine in diabetic patients compared to the audit standard of
Quality and Outcomes Framework (QOF) targets DM018, ${ }^{8}$ with the aim to reach the QOF target of $95 \%$ uptake as shown in Table 1 . The secondary objective was to identify which intervention increased the uptake of the flu vaccine by the greatest margin. The tertiary objective was to address any issues that arose as the audit progressed, for example, patient misconception about flu vaccine.

\section{Materials and methods Overview}

The setting of this audit was a general practice based in East London. The electronic clinical system, EMIS Web, is commonly used in primary care to provide efficient integrated health care. This was the primary source of data in this audit. EMIS Web was used to look at QOF targets and practice information. QOF targets were created to provide financial incentives to NHS services, promoting efficiency and overall improved patient care. QOF targets were used as the audit standard by which the code DM018 was used to identify those within the diabetic population that were yet to receive the vaccine. The audit was undertaken over a 7-week period from October 23, 2017, to December 11, 2017, and involved contacting patients on three different occasions and booking them into various flu jab clinics over that time period.

\section{Parent population}

The parent population was calculated using EMIS and the DM018 code for QOF targets. The inclusion criteria were all diabetic patients who had not received the influenza vaccine for 2017 and the exclusion criteria were all diabetic patients who had the vaccine at that given point in time and nondiabetic patients. The EMIS system is updated daily and an audit is run every day keeping the number of patients on the DM018 list up to date.

Table I Pre-audit QOF data, including QOF targets and current general practitioner practice percentages in the given population, for the code DMOI 8 (the percentage of patients, on the register, who have had influenza immunization)

\begin{tabular}{|l|l|l|l|l|}
\hline QOF code & Description & Population count, $\mathbf{n}$ & Population (\%) & Target \% \\
\hline DM017 & Patients on diabetic register & 417 & 4 & 0 \\
\hline DM002 & Diabetes and BP $\leq 150 / 90 \mathrm{~mm} / \mathrm{Hg}$ & 358 & 88 & 93 \\
\hline DM003 & Diabetes and BP $\leq 140 / 80 \mathrm{~mm} / \mathrm{Hg}$ & 275 & 69 & 78 \\
\hline DM004 & Diabetes and cholesterol $\leq 5.0 \mathrm{mmol} / \mathrm{L}$ & 291 & 76 & 75 \\
\hline DM006 & Diabetes and proteinuria or microalbuminuria & 24 & 83 & 97 \\
\hline DM007 & Diabetes and IFCC-HbAIc $\leq 59 \mathrm{mmol} / \mathrm{mol}$ & 242 & $6 I$ & 75 \\
\hline DM008 & Diabetes and IFCC-HbAIc $\leq 64 \mathrm{mmol} / \mathrm{mol}$ & 272 & 68 & 83 \\
\hline DM009 & Diabetes and IFCC-HbAIc $\leq 75 \mathrm{mmol} / \mathrm{mol}$ & $3 I \mathrm{I}$ & 92 \\
\hline DM0I2 & Diabetes and foot risk assessment in I2 months & 344 & 86 & 90 \\
\hline DM018 & Diabetes given Influenza Vaccine & 231 & 59 & 95 \\
\hline
\end{tabular}

Abbreviations: QOF, Quality and Outcomes Framework; HbAIC, glycosylated hemoglobin; IFCC, International Federation of Clinical Chemistry. 


\section{Methods of contacting patients}

1. Use the EMIS system to find the number of diabetic patients who have had the flu jab (23rd October).

2. Determine the number of diabetic patients who are yet to receive the flu vaccine.

3. Collate patients who fit inclusion and exclusion criteria to form a list.

4. Use patient list in conjunction with EMIS system to find patient phone number.

5. Call patients between 10:00 and 18:00 across a 2-week period (October 23, 2017, to November 6, 2017), using script (Box S1) and record outcome of call according to key (Box S2).

6. Call all patients for a second time who are still on the DM018 list according to system update that day (November 6, 2017), using script (Box S1); if there is no answer, then leave a voicemail, using script (Box S3); and record outcome of phone call using key (Box S2). Across a 2-week period (November 6, 2017, to November 20, 2017).

7. Email all patients still remaining on the DM018 list using EMIS system to find email, using script (Box S4) (November 27, 2017).

8. Record new data (updated DM018 list) after a 2-week period (November 27, 2017, to December 11, 2017).

All the data that were collected from EMIS and the outcomes of the interventions were inputted into a password-protected spreadsheet to be used later to analyze the results.

\section{Ethics}

Ethics approval was not needed for this project as it is a clinical audit and did not involve anything beyond the patients routine clinical management.

\section{Results}

The pre-audit information indicated a 95\% QOF target for the number of diabetic patients registered with the practice that needed to have the flu jab. The practice had successfully vaccinated $59 \%$ of these patients at the start of the audit (October 23, 2017), as shown in Table 1. Similarly, looking at the same data post-audit, the percentage of patients who had successfully received the vaccine in the diabetic population had increased to $77 \%$, as shown in Table 2. It should be noted that the value of $77 \%$ is recorded on the EMIS system; however, as a result of our data, we found that the actual percentage who have had the vaccine was $79 \%$, accounting for $2 \%$ who had the vaccine at their local chemist.

The data from 2016 (the previous year) where patient notification was not implemented showed an $8 \%$ increase in the uptake of the vaccine across the same 7 -week period. This accounts for the promotion of the influenza vaccine already done by the practice when patients attend their appointments. In comparison to this data, the data from 2017 show a $12 \%$ increase in the uptake of the vaccine that can be attributed to patient notification interventions over the 7 -week period.

\section{Intervention I}

The parent population of diabetic patients who were yet to have the flu vaccine came to a total of 393 patients. Of these patients five did not have any contact information and so could not be notified as part of interventions 1,2, or 3. Of the remaining 388, seven had incorrect telephone numbers and so could not be notified through interventions 1 and 2, as shown in Figure 1. This left 381 who satisfied the criteria and were called as part of intervention 1. Using the key (Box S2) for the outcomes, A had 17, B had 8, C had 222, D had 14, E had 95, F had 14, and G had 11. After this, data were

Table 2 Post-audit QOF data, including QOF targets and current general practitioner practice percentages in the given population, for the code DMOI8 (The percentage of patients, on the register, who have had influenza immunization)

\begin{tabular}{|l|l|l|l|l|}
\hline QOF Code & Description & Population count, $\mathbf{n}$ & Population (\%) & Target \% \\
\hline DM0I7 & Patients on diabetic register & 419 & 4 & 0 \\
\hline DM002 & Diabetes and BP $\leq 150 / 90 \mathrm{~mm} / \mathrm{Hg}$ & 350 & 86 & 93 \\
\hline DM003 & Diabetes and BP $\leq 140 / 80 \mathrm{~mm} / \mathrm{Hg}$ & 277 & 70 & 78 \\
\hline DM004 & Diabetes and cholesterol $\leq 5.0 \mathrm{mmol} / \mathrm{L}$ & 288 & 74 & 75 \\
\hline DM006 & Diabetes and proteinuria or microalbuminuria & 25 & 83 & 97 \\
\hline DM007 & Diabetes and IFCC-HbAIc $\leq 59 \mathrm{mmol} / \mathrm{mol}$ & 232 & 59 & 75 \\
\hline DM008 & Diabetes and IFCC-HbAIc $\leq 64 \mathrm{mmol} / \mathrm{mol}$ & 266 & 67 & 83 \\
\hline DM009 & Diabetes and IFCC-HbAIc $\leq 75 \mathrm{mmol} / \mathrm{mol}$ & $31 \mathrm{I}$ & 78 & 92 \\
\hline DM0I2 & Diabetes and foot risk assessment in I2 months & 344 & 87 & 90 \\
\hline DM0I8 & Diabetes given influenza vaccine & 305 & 77 & 95 \\
\hline
\end{tabular}

Notes: QOF codes and descriptions adapted from: DM018 (The percentage of patients, on the register, who have had influenza immunisation) Reference: Nhs.org. (2018). Summary of Changes to QOF. Available from: https://www.nhsemployers.org//media/Employers/Documents/Primary-care-contracts/QOF/2017-18/201718-Quality-andoutcomes-framework-summary-of-changes.pdf. Accessed November 9, 2018.

Abbreviations: QOF, Quality and Outcomes Framework; HbAIC, glycosylated hemoglobin; IFCC, International Federation of Clinical Chemistry. 


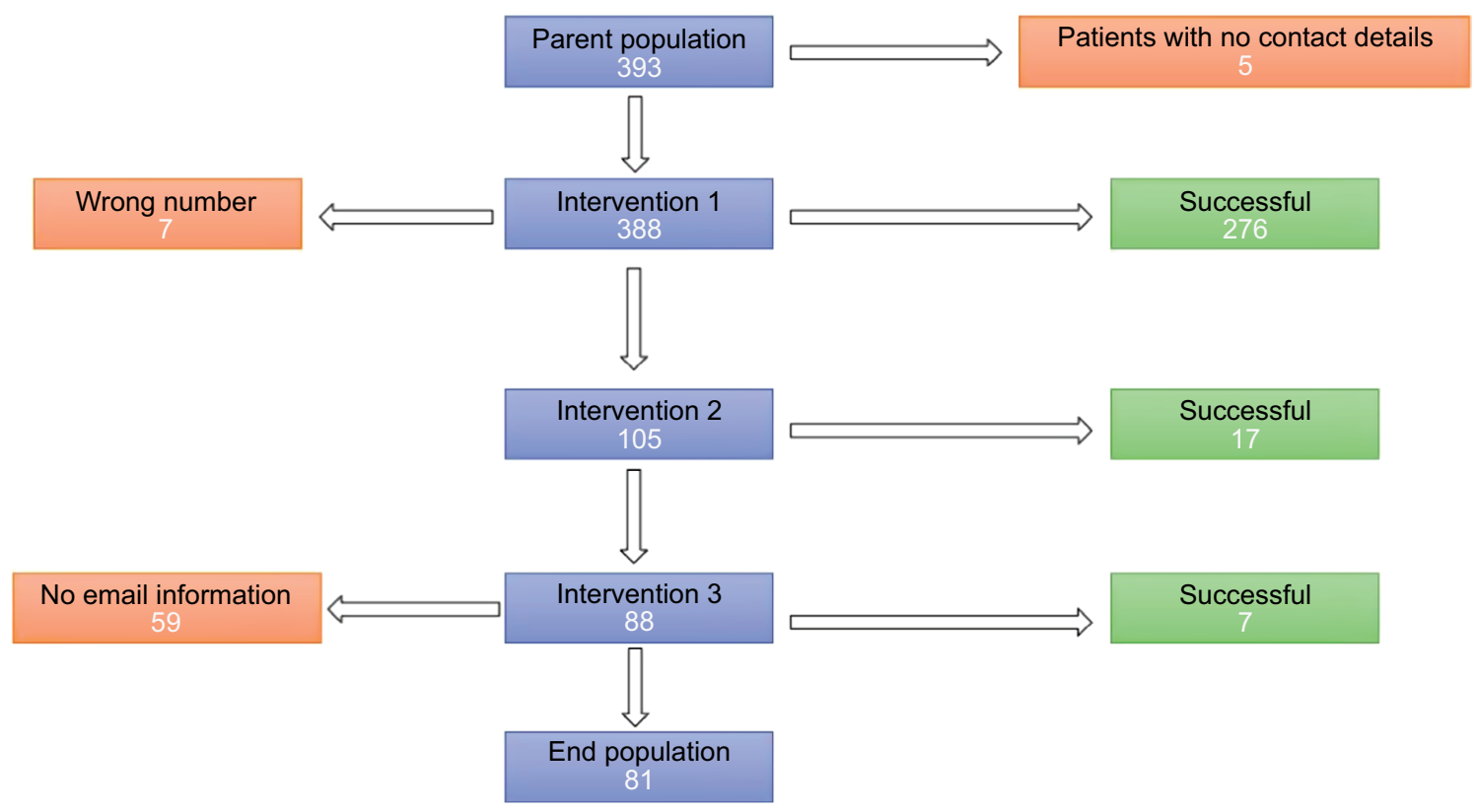

Figure I A broad overview of the patient count through the course of the audit.

Notes: Intervention I (first telephone call made to patients all patients); intervention 2 (second telephone call made to patients who still had not received the vaccine); and intervention 3 (email sent to remaining patients).

collected at the end of the 2-week period, 105 still required the flu vaccine and had not declined.

\section{Intervention 2}

The second intervention involved calling up the remaining 105 patients and recording outcomes of the call once again. This intervention was altered slightly, compared to the first, as a voicemail was left for patients who did not answer for the second time (if this was possible). Using the key (Box S2) for the outcomes, A had 0, B had 13, C had 38, D had 9, E had $37, \mathrm{~F}$ had 3 , and $\mathrm{G}$ had 5. At the end of the 2-week period, the data were collated and it was found that 88 patients were still in need of the flu vaccine and had not declined. After the first and second interventions, the various reasons for patients declining the flu vaccine were noted and compiled, as shown in Figure 2.

\section{Intervention 3}

Sending an email reminder about the flu vaccine was the third and final intervention. There were 88 patients remaining, of whom 29 had email addresses on the EMIS database. Therefore, those 29 were sent an email. After a 2 -week period, 7 out of the 29 emailed received the flu vaccine. The final results showed that 81 patients were still in need of the flu vaccine (December 11, 2017).

\section{Discussion}

A comparison between the pre-audit and post-audit figures show a $20 \%$ increase in the number of patients vaccinated

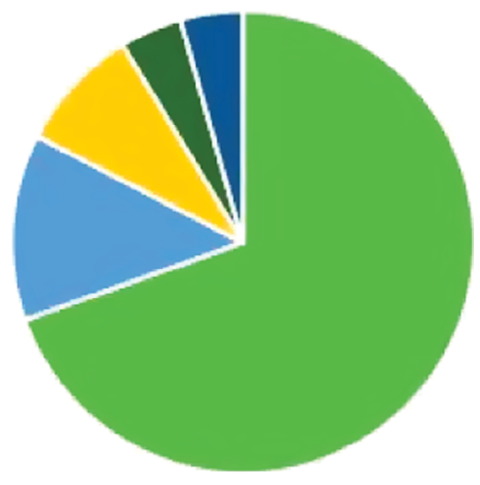

\footnotetext{
No reason

- Previous bad experience

Currently ill but will get it once well

- Not cold enough to get it done

- Traveling
}

Figure 2 Reasons patients declined the flu vaccine in intervention I (first telephone call made to patients) and intervention 2 (second telephone call made to patients). Nearly $70 \%$ chose to give no reason, $13 \%$ declined due to previous bad experience, $8 \%$ were ill but were insistent they would get the vaccine once well, $4 \%$ were traveling, and $4 \%$ believed it was not cold enough to get the flu vaccine yet.

against the flu in the diabetic population at this practice (Tables 1 and 2). Using the three different interventions amounted to this significant increase and highlighted various issues.

From the very beginning of the audit, it became clear that there would be some issues around the database and patient information. After identifying 393 diabetic patients who were yet to have the flu vaccine, 5 of the 393 were immediately 
ruled out as noncontactable due to lack of telephone numbers or email addresses on the database. Similarly, at the end of the first intervention, it became clear that 7 of the 388 patients had incorrect telephone numbers (Figure 1). This poses a wider issue around the collection of patient contact details and the fact that patients may be put at greater risk of health deprivation by missing out on reminders from primary care. This has the potential to be detrimental to their health, particularly in this patient group (diabetics) who require constant follow-up and checks to ensure optimum health.

Likewise, when it came to the third intervention, only $33 \%$ of the patients that still required the vaccine ( 88 patients) had their email addresses recorded on the system. This once again had a massive impact on the results. This intervention was the least time consuming but effective as it negated many patient factors that may have been a hindrance in interventions 1 and 2, such as time of day the call was made, patient availability, and patients response to an unknown caller.

After the first and second interventions, it was noted that several patients were down as having not received the vaccine but in actual fact, they had it done at their local chemist. It begs the question of whether there is a need for a more cohesive, transparent, and holistic relationship between general practitioner practices and other community healthcare services to improve efficiency.

A significant proportion of the patients contacted in interventions 1 and 2 declined the flu vaccine. When asked why, there were varying reasons given (Figure 2). Many chose not to give a reason; however, it became clear with the other patients that there were quite a few misconceptions about the vaccine - how it works, when best to have it, and the side effects.

Moreover, stratification of the patient population into high-risk and low-risk groups may have been beneficial in targeting patients who were most in need of the vaccine, that is, those patients above a certain age or with different treatment types. However, in order to have the most widereaching impact on the population, everyone who fell into the inclusion criteria were contacted.

Given the area of London the practice is based and the propensity diabetes has to people of ethnic backgrounds (Asian and Afro-Caribbean populations), it became clear that language was a barrier for several patients when seeking medical care. This made it difficult communicating the purpose of calls in interventions 1 and 2 and even more difficult when talking to a patient who wanted to decline the flu vaccine. Therefore, it would be useful to have healthcare professionals available to speak to patients in their language to get across the main message of patient notifications. It was interesting to note that throughout the winter months, there is a "Stay Well This Winter" national television campaign to promote the uptake of the flu vaccine. ${ }^{9}$ This has been translated into Hindi and aired on particular television channels to target a population with a high incidence of diabetes. It would be great to see more of these campaigns in other languages as well as being available to watch at primary care centers as this has the potential to significantly increase the flu vaccine in these patients.

This audit was conducted in an area of London with high levels of ethnic diversity, low rates of employment, and below-average income levels when compared to national figures. The age distribution in this area follows the distribution across the nation. ${ }^{10}$ Although, the various socioeconomic factors differ from those across the country, we postulate that the audit results can be generalized as diabetes is a disease that affects people across the country. Furthermore, with the higher rates of native English speakers nationwide, compared to the population in this area, an even greater uptake of the vaccine can be expected as this overcomes the language barrier faced in this intervention.

\section{Conclusion}

In conclusion, the use of patient notifying interventions had an undoubted impact on the number of patients who received the flu vaccine throughout the 7 -week period of the audit. A $12 \%$ increase in the uptake of the vaccine can be attributed to patient notification when comparing the data with the previous year. Further work needs to be done on the impact of patient notification including feedback from patients as to whether they believe it is effective and what the best modes of contacting them are.

\section{Acknowledgments}

We would like to thank the Royal Docks Medical Practice and Dr Jim Lawrie for allowing us to undertake this audit. A poster based on this work was presented at the Royal Society of Medicine 38th Primary Care Update; September 11-13, 2018; London, UK.

\section{Disclosure}

The authors report no conflicts of interest in this work.

\section{References}

1. Diabetes UK. Diabetes Prevalence 2016 (November 2016); 2016. Available from: https://www.diabetes.org.uk/professionals/positionstatements-reports/statistics/diabetes-prevalence-2016. Accessed December 13, 2017.

2. Diabetes.co.uk. How Many People Have Diabetes - Diabetes Prevalence Numbers; 2017. Available from: https://www.diabetes.co.uk/diabetesprevalence.html. Accessed December 13, 2017. 
3. Newhamccg.nhs.uk. Newham Clinical Commissioning Group Diabetes in Newham; 2017. Available from: http://www.newhamccg. nhs.uk/services/diabetes-in-newham.htm. Accessed December 14, 2017.

4. Casqueiro J, Casqueiro J, Alves CAlves C, Casqueiro J. Infections in patients with diabetes mellitus: a review of pathogenesis. Indian $J$ Endocrinol Metab. 2012;16(Suppl 1):27.

5. Peleg AY, Weerarathna T, Mccarthy JS, Davis TM. Common infections in diabetes: pathogenesis, management and relationship to glycaemic control. Diabetes Metab Res Rev. 2007;23(1):3-13.

6. Patient.info. Diabetes Mellitus. Information about Diabetes Mellitus (DM). Patient; 2017. Available from: https://patient.info/doctor/ diabetes-mellitus-pro. Accessed December 14, 2017.
7. Nhs.uk. How the Adult Flu Vaccine Works - Vaccinations - NHS Choices; 2017. Available from: https://www.nhs.uk/Conditions/vaccinations/Pages/how-flu-vaccine-works.aspx. Accessed December 14, 2017.

8. NHS. Summary of Changes to QOF 2017-18 - England; 2018. Available from: https://www.nhsemployers.org/-/media/Employers/Documents/ Primary-care-contracts/QOF/2017-18/201718-Quality-and-outcomesframework-summary-of-changes.pdf. Accessed November 9, 2018

9. Campaignresources.phe.gov.uk. Stay Well This Winter; 2017. Available from: https://campaignresources.phe.gov.uk/resources/campaigns/34stay-well-this-winter-/resources. Accessed December 14, 2017.

10. Aston-mansfield.org.uk. Newham Key Statistics 2017; 2017. Available from: https://www.aston-mansfield.org.uk/wp-content/themes/aston_mansfield/uploads/Newham_Statistics_2017.pdf. Accessed August 29, 2017. 


\section{Supplementary materials}

Box SI Script used when on the telephone phone to patients

Hello,

I am calling on behalf of Dr Jim Lawrie from the Royal Docks Medical Practice.

Records show you are due for your annual flu jab.

Would you be interested in booking an appointment to come in sometime this week or next to have it done?

Will only take 5-10 minutes.

If not may I ask why?

Thank you

Box S2 Keys used to identify and record outcome of telephone call

A - Already booked an appointment for the flu jab

B - Already got a diabetic-related appointment booked in $<2$ weeks time

C - Already had the flu jab done

D - Declined the flu jab

E - Did not answer

$\mathrm{F}$ - Flu jab booked with us

G - Would like to book an appointment at a later date
Box S3 Script for the voicemail that was used when a patient was reached on the telephone

Hello, I am calling for Mr/Ms X.

I am calling on behalf of Dr Jim Lawrie from the Royal Docks Medical

Practice.

Records show you are due for your annual flu jab.

Please call the practice to book this in as soon as possible.

Thank you

Box S4 Email that was sent out to patients

Good morning,

I'm contacting you on behalf of Dr Jim Lawrie of the Royal Docks GP practice. Our records show that you have not yet received your flu jab this year. Please feel free to call us to book your appointment. For more information on the flu jab and its benefits in diabetics please visit: http://www.diabetes.co.uk/flu-vaccination.html Best wishes,

Royal Docks Medical Practice

Abbreviation: GP, general practitioner.

\section{Clinical Audit}

\section{Publish your work in this journal}

Clinical Audit is an international, peer-reviewed, open access journal focusing on the processes and outcomes of clinical audit in any area of healthcare. All aspects of patient care are addressed within the journal and practitioners from all disciplines are invited to submit their work. Areas covered include: Publication of audits; How an audit has changed practice;

\section{Dovepress}

Practical tips on how to do audits and to avoid pitfalls; How audits have changed patient care; Calls and justifications for new audits. The manuscript management system is completely online and includes a very quick and fair peer-review system, which is all easy to use. Visit http://www.dovepress. com/testimonials.php to read real quotes from published authors. 